\title{
Light-Front QCD Hamiltonian Dynamics and Constituent Quark Picture in Exclusive Processes
}

\author{
Chueng-Ryong $\mathrm{Ji}^{\mathrm{a} *}$ and Ho-Meoyng Choi ${ }^{\mathrm{b} \dagger}$

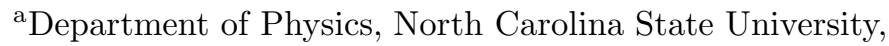 \\ Raleigh, NC 27695-8202, U.S.A \\ ${ }^{\mathrm{b}}$ Department of Physics, Carnegie Mellon University, \\ Pittsburgh, PA 15213, U.S.A
}

After reviewing a connection between quantum chromodynamics and contituent quark model pictures in the light-front quantization with some comparison and contrast to the ordinary equal-time bridge a la BogoliubovValatin transformation, we discuss some newer development of the light-front quark model phenomenology in exclusive processes including the embedded state. The skewed parton distribution appears to be a good testing ground for our new effective treatment of the light-front nonvalence contributions in timelike exclusive processes.

One of the most puzzling features in hadron physics is the connection between the two fundamentally different pictures of hadronic matter, i.e. the quantum chromodynamics (QCD) based on a covariant non-Abelian quantum field theory and the constituent quark model (CQM) closely related to experimental observations. While the QCD has a complicate nonperturbative vacuum structure manifested by the color confinement and the dynamical breaking of the chiral symmetry, the CQM is mostly built on a rather simple vacuum. Because of the bound state problem inherent in the hadron physics, the Hamiltonian approach based on an equal-time formulation is often used to remove the complications from the relative time degrees of freedom among the constituents. In this respect, both the ordinary time $t$ and the light-front(LF) time $\tau=t+z / c$ are by far the most popular choices for an equal-time formulation. However, these two choices render quite different pictures for the bridge between QCD and CQM.

In the equal- $t$ approach, one needs to transform a complicate QCD vacuum to a rather simple CQM vacuum. To do this, people have of-

\footnotetext{
*ji@ncsu.edu

†homeoyng@andrew.cmu.edu
}

ten utilized the Bardeen-Cooper-Schrieffer(BCS) type Bogoliubov-Valatin (BV) transformation [13. With this transformation, one can get a coherent vacuum for quasiparticle constituents and the mass gap equation providing a relation beween the current quark mass and the consituent quark mass. The QCD Hamiltonian $H_{Q C D}$ in the Coulomb gauge can be split into $H_{0}$ describing the ball park of the physical system and $H_{I}$ being the rest of the Hamiltonian that can give small perturbative corrections. This splitting can be done by adding and subtracting a phenomenological Hamiltonian $H_{\text {phen }}$ to the kinetic energy part $K$ and the interaction part $H_{Q C D}^{I}$, respectively, i.e.

$$
\begin{aligned}
H_{Q C D} & =\left(K+H_{p h e n}\right)+\left(H_{Q C D}^{I}-H_{p h e n}\right)(1) \\
& =H_{0}+H_{I} .
\end{aligned}
$$

While $H_{\text {phen }}$ is often given by the confining (e.g. linear) potental, the residual interaction $H_{I}$ is ususally taken as the canonical QCD interaction Hamiltonian at the cutoff scale $\Lambda$ infinite; $H_{I} \approx H_{\text {can }}^{I}(\Lambda \rightarrow \infty)$. However, in order to find a low energy effective Hamiltonian, one needs to set $\Lambda$ finite and lower it to the scale of the effective Hamiltonian that one wants to find. Introducing a finite $\Lambda$ breaks the symmetry of the orginal 
Hamiltonian and to recover it one needs a counter term $H_{C T}(\Lambda)$. Lowering $\Lambda$ generates the part of effective Hamiltonian $H_{g e n}(\Lambda)$ that compensates the physics between the two $\Lambda$ values before and after lowering it. Both $H_{C T}(\Lambda)$ and $H_{g e n}(\Lambda)$ can be found by either a similarity renormalization procedure [2] or a flow equation method [3].

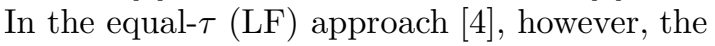
procedure of connecting between QCD and CQM may be drastically different from the equal- $t$ case since the vacuum at equal $\tau$ has a dramatic difference compare to the vacuum at equal $t$. For the particle which has the mass $m$ and the fourmomentum $k=\left(k^{0}, k^{1}, k^{2}, k^{3}\right)$, the relativistic energy-momentum relation at equal $\tau$ is given by

$k^{-}=\frac{\mathbf{k}_{\perp i}^{2}+m^{2}}{k^{+}}$,

where the LF energy conjugate to $\tau$ is given by $k^{-}=k^{0}-k^{3}$ and the LF momenta $k^{+}=k^{0}+k^{3}$ and $\mathbf{k}_{\perp}=\left(k^{1}, k^{2}\right)$ are orthogonal to $k^{-}$and form the LF three-momentum $\underline{k}=\left(k^{+}, \mathbf{k}_{\perp}\right)$. The rational relation given by Eq.(2) provides a remarkable feature to the LF vacuum, namely, the Fock state vacuum is an eigenstate of the full Hamiltonian. Consequently, all bare quanta in hadronic Fock states are associated with the hadron and none are disconnected elements of the vacuum. This leads to a relatively simple vacuum structure in QCD. There is no spontaneous creation of massive fermions in the LF quantized vacuum. Thus, one can immediately obtain a constituent-type picture, in which all partons in a hadronic state are connected directly to the hadron instead of being simply disconnected excitations (or vacuum fluctuations) in a complicated medium. Nevertheless, one needs to cutoff the zero-modes [5] corresponding to the degrees of freedom with $k^{+} \rightarrow 0$ to obtain a salient CQM picture because the zeromode fluctuations are still possible in the LF vacuum. The cutoff of the zero-modes introduces the mass scale of the constituent quark as well as the counter term that can generate a non zero amplitude of particle creation and is therefore a possible source for the features associated with a nontrivial vacuum structure including confinement and spontaneous symmetry breaking.

Recently, the flow equation method of continu- ous unitary transformations was used to eliminate the minimal quark-gluon interaction in the LF $H_{Q C D}$ [6]. Dividing the complete Fock space into two components, a tractable $P$ subspace spanned by states with a small number of quanta and the remainder $Q=1-P$, one can get a Hamiltonian matrix to be diagonalized of the form

$H=\left(\begin{array}{ll}P H P & P H Q \\ Q H P & Q H Q\end{array}\right)$.

The flow equation method leads to block diagonalize this matrix yielding the effective Hamiltonian

$H_{e f f}=\left(\begin{array}{cc}P H_{e f f} P & 0 \\ 0 & Q H_{e f f} Q\end{array}\right)$.

One can then separately diagonalize the two blocks which are now uncoupled. The coupled differential equations in the two lowest Fock sectors correspond to the renormalization of the LF gluon mass and the generation of an effective quark-antiquark (as well as gluon-gluon) interaction. From these, a more singular $1 / q^{4}$ behavior for the quark and gluon effective interactions at small gluon momenta can be obtained [6]. Therefore, one can catch a glimpse of support from the LF QCD to the LF CQM which we call LF quark model (LFQM) [7].

Indeed, there has been a significant progress in describing the meson properties with the LFQM for the spacelike region [8]. Again, the success of this model hinges upon the simplicity of LF vacuum. The complicated nontrivial vacuum effect from the zero-modes has been traded off by the constituent quark masses. Moreover, the Drell-Yan-West $\left(q^{+}=q^{0}+q^{3}=0\right)$ frame in the LF quantization provided an effective formulation for the calculation of various form factors in the spacelike momentum transfer region $q^{2}=-Q^{2}<0$ [9]. As an example, only the valence diagram shown in Fig. 1(a) is needed in $q^{+}=0$ frame when the "good" components of the current, $j^{+}$and $j_{\perp}=\left(j_{x}, j_{y}\right)$, are used for the spacelike electromagnetic form factor calculation of pseudoscalar mesons. Successful LFQM description of various hadron form factors can be found in the literatures [7, 10]. 


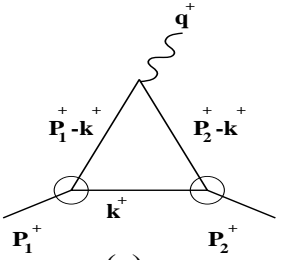

(a)

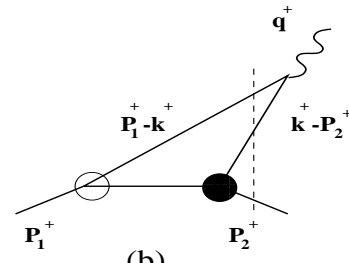

(b)
Figure 1. (a) The usual light-front valence diagram and (b) the nonvalence(pair-creation) diagram. The vertical dashed line in (b) indicates the energy-denominator for the nonvalence contributions.

However, not all is well. The timelike $\left(q^{2}>0\right)$ form factor analysis in the LFQM has been hindered by the fact that $q^{+}=0$ frame is defined only in the spacelike region $\left(q^{2}=q^{+} q^{-}-q_{\perp}^{2}<0\right)$. While the $q^{+} \neq 0$ frame can be used in principle to compute the timelike form factors, it is inevitable (if $q^{+} \neq 0$ ) to encounter the nonvalence diagram arising from the quark-antiquark pair creation (so called "Z-graph"). For example, the nonvalence diagram in the case of semileptonic meson decays is shown in Fig. 1(b). The main source of the difficulty, however, in calculating the nonvalence diagram (see Fig. 1(b)) is the lack of information on the embedded state represented by the black blob which should contrast with the white blob representing the usual $\mathrm{LF}$ valence wave function.

Fortunately, we've recently came up with an effective way of handling the nonvalence contribution [11]. Our aim of new treatment was to make the program suitable for the CQM phenomenology specific to the low momentum transfer processes. The key of our method is the link between the non-wave-function vertex (black blob) and the ordinary LF wave function (white blob) as shown in Fig. 2, i.e.,

$$
\begin{aligned}
& \left(M^{2}-M_{0}^{\prime 2}\right) \Psi^{\prime}\left(x_{i}, \mathbf{k}_{\perp i}\right) \\
& =\int[d y]\left[d^{2} \mathbf{l}_{\perp}\right] K\left(x_{i}, \mathbf{k}_{\perp i} ; y_{j}, \mathbf{l}_{\perp j}\right) \Psi\left(y_{j}, \mathbf{l}_{\perp j}\right),
\end{aligned}
$$

where $M$ is the mass of outgoing meson and $M_{0}^{\prime 2}=\left(m_{1}^{2}+\mathbf{k}_{\perp 1}^{2}\right) / x_{1}-\left(m_{2}^{2}+\mathbf{k}_{\perp 2}^{2}\right) /\left(-x_{2}\right)$ with $x_{1}=1-x_{2}>1$ due to the kinematics of the non-wave-function vertex.

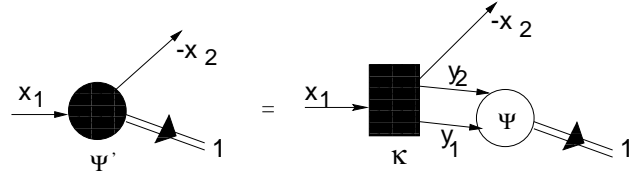

Figure 2. Non-wave-function vertex(black blob) linked to an ordinary LF wave function(white blob).

To discuss further details of our method [1], we formulate the pion form factor in terms of the off-forward parton distribution functions, the so called "skewed parton distributions (SPDs)" that are the generalization of the ordinary (forward) distribution functions [12]. A well-known and practical example of SPDs as a nonperturbative information entering the LF dominated hard scattering processes is the deeply virtual Compton scattering (DVCS) $\gamma^{*} p \rightarrow \gamma p$ for large initial photon virtuality $Q^{2}$ and small $t$ region, which can be factorized into a hard photon-parton and a skewed parton distribution $13-15]$. Since the usual local photon vertex in the pion form factor analysis is replaced by a nonlocal operator of the SPDs, one can explore new physics.

In the LF coordinates, the SPDs are in general functions of the longitudinal momentum fraction variable $x$, the skewedness parameter $\xi=$ $\left(P-P^{\prime}\right)^{+} / P^{+}$measuring asymmetry between initial $(P)$ and final $\left(P^{\prime}\right)$ hadron state momenta, and the squared momentum transfer $t$. Analogous to the pion electromagnetic (EM) form factor calculation

$J^{+}(0) \equiv\left\langle P^{\prime}\left|\bar{\psi}(0) \gamma^{+} \psi(0)\right| P\right\rangle=F_{\pi}(t)\left(P+P^{\prime}\right)^{+},(6)$

we define the $\operatorname{SPD} \mathcal{F}_{\pi}(\xi, x, t)$ of a pion by

$$
\begin{aligned}
\mathcal{J}^{+} & \equiv \int \frac{d z^{-}}{4 \pi} e^{i x P^{+} z^{-} / 2}\left\langle P^{\prime}\left|\bar{\psi}(0) \gamma^{+} \psi(z)\right| P\right\rangle \\
& =\mathcal{F}_{\pi}(\xi, x, t)\left(P+P^{\prime}\right)^{+}
\end{aligned}
$$

where $z=\left(z^{+}, z^{-}, \mathbf{z}_{\perp}\right)$ in a LF representation and $z^{+}=\mathbf{z}_{\perp}=0$. The SPDs display characteristics of the ordinary(forward) quark distribution in the limit of $\xi \rightarrow 0$ and $t \rightarrow 0$, on the other hand, the first moment of the SPDs is related to 


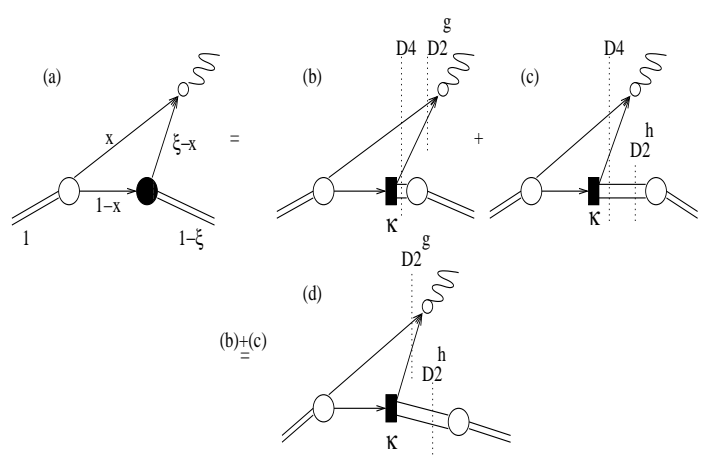

Figure 3. Effective treatment of the LF nonvalence amplitude.

the form factor by the following sum rules [14, 15]:

$$
\int_{0}^{1} d x \mathcal{F}_{\pi}(\xi, x, t)=F_{\pi}(t)
$$

where $\mathcal{F}_{\pi}(\xi, x, t)=e_{u} \mathcal{F}_{\pi}^{u}(\xi, x, t)-e_{d} \mathcal{F}_{\pi}^{\bar{d}}(\xi, x, t)$ and we assume isospin symmetry $\left(m_{u}=m_{\bar{d}}\right)$ so that $\mathcal{F}_{\pi}^{u}(\xi, x, t)=\mathcal{F}_{\pi}^{d}(\xi, x, t)$. Note that Eq. (8) is independent of $\xi$, which provides important constraints on any model calculation of the SPDs.

Considering the quark-meson and quark-gauge boson vertices together, we also find that the four-body energy denominator $\left(D_{4}\right)$ appearing in Fig. 3 is absent due to the sum of two possible diagrams in the LF time-ordering (see Figs. 3(b) and $(\mathrm{c})$ ). Summing over the two time-ordered diagrams Figs. 3 (b) and (c), one can easily find the following identity, $1 / D_{4} D_{2}^{g}+1 / D_{4} D_{2}^{h}=1 / D_{2}^{g} D_{2}^{h}$, which removes the complicate four-body energy denominator term. We thus obtain the amplitude corresponding to the nonvalence contribution in terms of ordinary LF wave functions of hadron and gauge boson as shown in Fig. 3(d). This method, however, requires the relevant operator $\mathcal{K}\left(x, \mathbf{k}_{\perp} ; y, \mathbf{l}_{\perp}\right)$ which is in general dependent on the involved momenta connecting the one-body to three-body sector as depicted in Fig. 2. The details of the valence and nonvalence contributions to the SPDs of the pion in LFQM can be found in Ref. [12]. While the relevant operator $\mathcal{K}$ is in general dependent on all internal momenta $\left(x, \mathbf{k}_{\perp} ; y, \mathbf{l}_{\perp}\right)$, the integral of $\mathcal{K}$ over $y$ and $\mathbf{l}_{\perp}$ in the nonvalence contribution, which we define as $G_{\pi} \equiv \int[d y]\left[d^{2} \mathbf{l}_{\perp}\right] \mathcal{K}\left(x, \mathbf{k}_{\perp} ; y, \mathbf{l}_{\perp}\right) \chi_{(2 \rightarrow 2)}\left(y, \mathbf{l}_{\perp}\right)$, depends only on $x$ and $\mathbf{k}_{\perp}$. Approximating $G_{\pi}$ as a constant has been tested in our previous exclusive semileptonic decay processes [11] and proved to be a good approxiamtion at least for small momentum transfer region. The validity of this approximation can be checked by fixing the constant $G_{\pi}$ by the sum rule expressed in terms of $\mathcal{F}_{\pi}^{v a l}$ and $\mathcal{F}_{\pi}^{n v}$ as

$F_{\pi}(t)=\int_{\xi}^{1} d x \mathcal{F}_{\pi}^{v a l}(\xi, x, t)+\int_{0}^{\xi} d x \mathcal{F}_{\pi}^{n v}(\xi, x, t),(9)$

for given $-t$. We note that Eq. (9) is used as a constraint on the frame-independence of our model.

In Fig. 目, we show the $\xi$-dependence of $G_{\pi}$ for different $-t$-values, i.e. $-t=0$ (diamond), 0.2 (black circle), 0.5 (white circle), and 1.0 (black square) $\left[\mathrm{GeV}^{2}\right]$, respectively. As one can see in Fig. 1, $G_{\pi}$ shows approximately constant behavior for $\xi>0.1$ at given small $-t$. It is not surprising to see that $G_{\pi}$ becomes very large as $\xi \rightarrow 0$, because $\mathcal{F}_{\pi}^{n v}$ has the form of $\mathcal{F}_{\pi}^{n v}=G_{\pi} \times \int_{0}^{\xi} \ldots$ and the integral vanishes while a small but nonzero contribution persists in $\mathcal{F}_{\pi}^{n v}$. However, this does not cause a significant error in our $G_{\pi}$ constant approximation because the nonvalence contribution in the very small $\xi$ region is highly suppressed. Therefore, the results are consistent with an almost constant value for $G_{\pi}$ at least for small $-t$. In principle, we can obtain the SPDs in a frame-independent way by using the true values of $G_{\pi}$ as shown in Fig. Af for given $(\xi, t)$.

In this talk, we reviewed the connection between QCD and CQM pictures in the LF quantization with a comparison and contrast to the ordinary equal-t approach. We then discussed the newer development of LFQM phenomenology in exclusive processes. The SPD appears to be a good testing ground of our new effective treatment in timelike exclusive processes. We investigated the SPDs of the pion for small momentum transfer $\left(-t \leq 1 \mathrm{GeV}^{2}\right)$ region in the LF quark model. Since the LF nonvalence contributions to the SPDs of the pion are large especially at small momentum transfer region as shown in Ref. [12], it is very crucial to take them into account to 


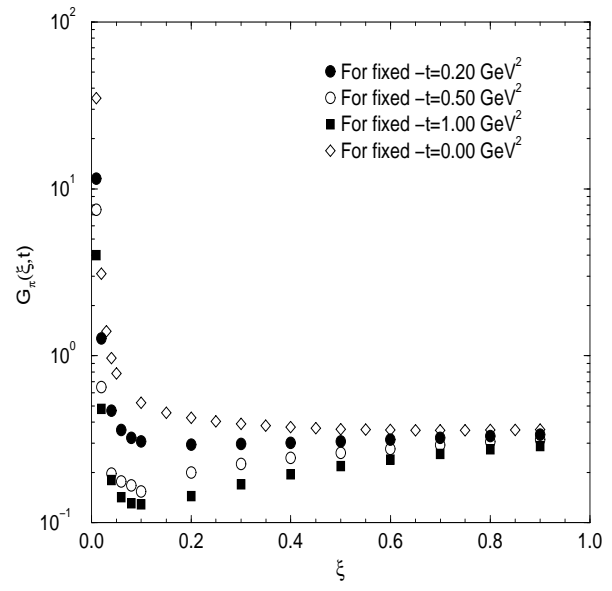

Figure 4 . The $\xi$-dependence of $G_{\pi}$ for different momentum transfers $-t=0$ (diamond), 0.2 (black circle), 0.5 (white circle), and 1 (black square) $\left[\mathrm{GeV}^{2}\right]$, respectively.

guarantee the frame-independence of the model. Applying our effective treatment [11], we express $\mathcal{F}_{\pi}^{n v}$ in terms of ordinary LF wave functions of a gauge boson and a hadron and calculate this nonvalence contribution numerically. The reliability of our constant approximation was checked by examining the frame-independence of our numerical results using the sum rule given by Eq. (9), i.e. the exact results of $\mathcal{F}_{\pi}(\xi, x, t)$ and $F_{\pi}(t)$ obtained from the true values of $G_{\pi}$ given by Fig. 4 were compared with those obtained from our single averge value of $G_{\pi}=0.32$ for all $(\xi, t)$. The numerical results of our constant $G_{\pi}$ prescription have shown definite improvement (better than 90 $\%$ accuracy for $\xi \lesssim 0.9$ ) to restore the frameindependence of our model (see Ref. [12] for details) and seemed to be a quite reliable approximation. However, we note that there is an obvious $t$-dependence for $G_{\pi}$, which leaves a room for more improvement of our model. Consideration of the kernel $\mathcal{K}$ in the gauge boson sector and the more realistic gauge boson wavefunction is underway.

\section{Acknowledgements}

We thank to the organizers of this workshop.
This work was supported in part by the US DOE under grant No. DE-FG02-96ER40947 and by the NSF grant PHY-00070888 and INT-9906384. The North Carolina Supercomputing Center and the National Energy Research Scientific Computer Center are also acknowledged for the grant of Cray time.

\section{REFERENCES}

1. A.P. Szczepaniak, E.S. Swanson, C.-R. Ji and S.R. Cotanch, Phys. Rev. Lett. 76, 2011 (1996).

2. D.G. Robertson, A.P. Szczepaniak, E.S. Swanson, C.-R. Ji and S.R. Cotanch, Phys. Rev. D 59, 074019 (1999).

3. E.L. Gubankova, C.-R. Ji and S.R. Cotanch, Phys. Rev. D 62, 074001 (2000).

4. S.J. Brodsky, H.-C. Pauli and S.S. Pinsky, Phys. Rept. 301, 299 (1998).

5. H.-M. Choi, and C.-R. Ji, Phys. Rev. D 58, 071901 (1998).

6. E.L. Gubankova, C.-R. Ji and S.R. Cotanch, Phys. Rev. D 62, 125012 (2000).

7. H.-M. Choi and C.-R. Ji, Nucl. Phys. A 618, 291 (1997); Phys. Rev. D 56, 6010 (1997); Phys. Lett. B 460, 461 (1999); Phys. Rev. D 59, 074015 (1999).

8. See H.-M. Choi, L.S. Kisslinger and C.-R. Ji in this Proceedings.

9. G.P. Lepage and S.J. Brodsky, Phys. Rev. D 22, 2157 (1980).

10. P.L. Chung, F. Coester and W.N. Polyzou, Phys. Lett. B 205, 545 (1988); W. Jaus, Phys. Rev. D 44, 2815 (1991); F. Cardarelli et al., Phys. Lett. B 332, 1 (1994); Phys. Rev. D 53, 6682 (1996).

11. C.-R. Ji and H.-M. Choi, Phys. Lett. B 513, 330 (2001).

12. H.-M. Choi, C.-R. Ji and L.S. Kisslinger, Phys. Rev. D 64, 093006 (2001)

13. D. Müller, D. Robaschik, B. Geyer, F. M. Dittes, J. Hořejši, Fortsch. Phys. 42, 101 (1994).

14. X. Ji, Phys. Rev. Lett. 78, 610 (1997); Phys. Rev. D 55, 7114 (1997).

15. A. V. Radyushkin, Phys. Rev. D 56, 5524 (1997). 